\title{
Differential Polarization of Spin and Charge Density in Substituted Phenoxy Radicals
}

\author{
Richard J. Fehir, Jr. and James K. McCusker* \\ Contribution from the Department of Chemistry, Michigan State University, East Lansing, Michigan 48824
}

Received: June 5, 2009

\begin{abstract}
We have carried out a theoretical study of a series of para-substituted phenoxy radicals in an effort to understand the factors influencing spin and charge density distribution in open-shell systems. The calculations reveal that the distribution of spin and charge are not correlated: cases were found for which spin and charge move together, whereas for other substituents the two quantities exhibit spatially distinct intramolecular polarizations. Charge density variations across the series were found to correlate well with both the Hammett $\left(\sigma_{\mathrm{p}}\right)$ and Hammett-Brown $\left({\sigma_{\mathrm{p}}}^{+}\right)$constants for each substituent, indicating that inductive and/or resonance effects are primarily responsible for the polarization of charge within the molecule. In contrast, the distribution of unpaired spin density could not be adequately accounted for using any of the typical Hammett-type spin delocalization constants cited in the literature. We uncovered an empirical correlation between the polarization of spin density and the $\alpha-H O M O-\alpha-L U M O$ gap of the substituted phenoxy radicals: this led to the development of a simple model based on a three-electron, two-orbital bonding scheme in which mixing between the HOMO of the substituent and the SOMO of the phenoxy moiety serves to define the nature and extent of unpaired spin polarization throughout the molecule. This analysis yielded a correlation coefficient of $r>0.97$ for the 15 substituents examined in the study; spin polarization effects in compounds that exhibited the greatest deviation from this correlation could also be readily explained within the context of the model. The underlying reason for the ability to differentially polarize spin and charge likely stems from the fact that net unpaired spin density is completely carried by the unpaired electron (and thus tracks the spatial characteristics of the SOMO), whereas charge density reflects the behavior of all of the electrons of the system. These results could have implications in the field of molecular magnetism, suggesting a means for synthetically tuning the magnitude of intramolecular exchange interactions, as well as providing guidance for the design of catalysts for radical-radical coupling reactions.
\end{abstract}

\section{Introduction}

Spin and charge represent two of the most fundamental physical properties in nature. Indeed, the role of these quantities in defining the electronic structures of atoms and molecules serves to underscore their importance in virtually all areas of molecular science. From relatively simple ideas such as nucleophilicity to more advanced concepts involving the emerging field of spintronics, ${ }^{1-3}$ the interplay between these two related but distinct aspects of electronic structure represents an intriguing aspect of the chemistry of open-shell systems.

Many of our expectations concerning charge distribution in molecular systems are based on the concept of electronegativity. This simple yet fundamental idea provides the foundation for envisioning the polarization of charge in response to compositional changes in a molecule. The correlation of experimental observables with the various Hammett-type relations that have been developed over the years has helped to put these ideas on a more quantitative (though still largely empirical) basis. ${ }^{4-8}$ In so doing, the physical-organic community in particular has been able to develop tremendous physical and mechanistic insights into the cause-and-effect relationship between charge polarization and chemical reactivity. ${ }^{9-13}$

A comparable level of understanding of the effect of spin on chemical reactivity suffers in part from the lack of a similarly intuitive counterpart to electronegativity for spin polarization. Experimental techniques for measuring the distribution of

\footnotetext{
* To whom correspondence should be addressed.
}

unpaired spin density, most notably EPR and ENDOR spectroscopies, have contributed greatly toward describing spin polarization in a wide range of chemical systems. The development of spin-unrestricted formalisms in density functional theory has also emerged as a powerful computational tool, particularly with regard to large molecules. ${ }^{14-16}$ Such calculations can provide a description of unpaired spin polarization and therefore a basis for correlating quantum-mechanical descriptions of systems with experimental data that directly ${ }^{17,18}$ or indirectly ${ }^{19-21}$ probe the spatial distribution of unpaired spin. In related work, the question of local spin and spin distribution in molecules has been considered in detail by Reiher, ${ }^{22}$ while Lecomte and co-workers have pioneered the use of high-resolution electron density maps to probe spin-spin interaction mechanisms in a variety of systems based on a detailed analysis of electron density distributions. ${ }^{23}$ Both of these efforts represent significant first steps toward making the connection between charge and spin density and assessing its impact on the physical and chemical properties of molecules.

In the field of magnetism, from which our interest in this issue originates, spin and charge play very different roles. For example, in inorganic-organic hybrid systems (e.g., metalsemiquinone complexes), ${ }^{24-27}$ large formation constants will generally be favored when the donor atom from the ligand possesses negative charge density sufficient for it to act as a strong Lewis base toward the metal ion. In this context, the charge density dictates, to a large extent, the stability of the adduct being formed. The magnitude of the exchange interaction between the paramagnetic constituents will reflect several 
SCHEME 1: Resonance Structures for the Phenoxy Radical

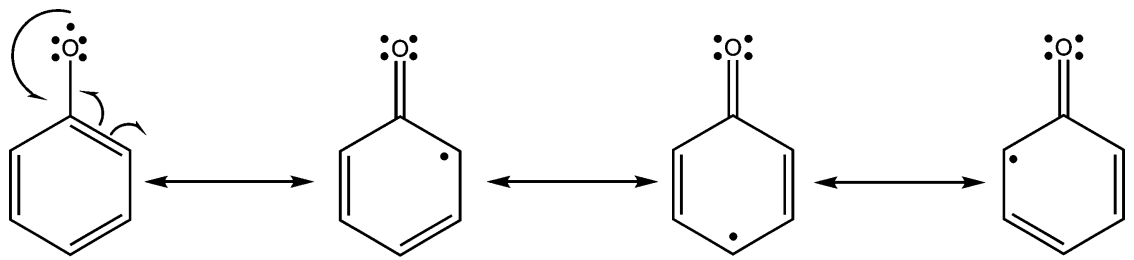

variables, but significant among these is the spatial distribution of unpaired spin density. All other factors being equal, ${ }^{28}$ polarization of unpaired spin density toward (or away) from the ligating atoms will increase (or decrease) the magnitude of electronic coupling. The notion of altering the magnetic properties of molecules through chemical means has been addressed both computationally ${ }^{29-32}$ and experimentally. ${ }^{33-35}$ Recent work by Shultz and co-workers, in particular, has clearly demonstrated that spin delocalization contributes to the attenuation of exchange interactions in bridged polynuclear systems. ${ }^{35-37}$ In other work, this team has provided examples of metalsemiquinone complexes in which changes in ring substituents give rise to variations in the magnitude of intramolecular spin exchange. ${ }^{38}$ Clearly, the ability to independently manipulate charge and spin density within a molecule has significant potential in terms of tailoring the thermodynamic as well as electronic properties of chemical systems.

At first glance, it would seem that spin and charge density should move in concert: since electrons carry both charge and spin, it seems reasonable that their polarizations should track each other. A case in point is the phenoxy radical, several resonance structures for which are shown in Scheme 1. We can see that the unpaired electron can be localized on the oxygen atom as well as the ortho and para positions on the ring. EPR data have confirmed that a significant portion of the spin density is in fact localized at the ortho and para carbon atoms. In addition, ${ }^{17} \mathrm{O}$ hyperfine coupling constants of 2,4,6-tri-t-butylphenoxy and 2,4,6-triphenylphenoxy radicals indicate that the spin density at the oxygen atom is approximately equal to the spin density at the ortho carbons, a result that is wholly consistent with this picture. ${ }^{39-42}$ Previous computational studies by various DFT methods support these findings in that a substantial fraction of the spin density was found to be associated with the oxygen atom. ${ }^{43,44}$ Not surprisingly, these calculations also revealed that the oxygen carries most of the negative charge density. The combined experimental and theoretical data therefore show that the spin and charge density in the (unsubstituted) phenoxy radical are both polarized in the same direction, i.e., toward the oxygen atom. This case thus conforms to our initial expectations.

Contrast the above observations with what has been described for the ketyl radical. The resonance structures in Scheme 2 show that the spin and/or charge density can be accommodated at either the carbonyl carbon or the oxygen atom. DFT calculations have shown that the charge density is largely localized on the oxygen atom, ${ }^{45}$ as expected. The EPR spectrum of the benzophenone ketyl radical exhibits ${ }^{17} \mathrm{O}$ and ${ }^{13} \mathrm{C}$ hyperfine coupling constants consistent with the spin density on both atoms of the

SCHEME 2: Resonance Structures for the Ketyl Radical<smiles>[R]C([O+])=[O+]CC</smiles>

carbonyl unit, however, the spin density was shown to be more highly localized at the carbon atom. ${ }^{46-48}$ DFT calculations yielded similar results.

These anecdotal results provide a convenient backdrop for the central question of this investigation: is it possible to design molecules in which spin and charge density can be independently manipulated? To examine this issue, we have carried out a computational study of substituted phenoxy radicals. As alluded to above, this system has been examined in some detail by other workers; the fact that spin and charge do not necessarily correlate is evident in the results of $\mathrm{Wu}$ and Lai in particular, ${ }^{44}$ however, the underlying reason for this observation has not been elucidated. In the course of analyzing our results, we have discovered a previously unrecognized correlation between spin polarization and electronic structure which we believe not only supports the notion of differential polarization of spin and charge, but suggests that the potential exists for the independent manipulation of these two variables through chemical means as a mechanism for tailoring the physical properties of molecules.

\section{Experimental Section}

General Methods. All self-consistent field Hartree-Fock and density functional calculations were carried out using Gaussian 98. ${ }^{49}$ Both the BLYP and B3LYP functionals were used in the calculations. The BLYP functional contains the gradient corrected Becke $(\mathrm{B})^{50}$ exchange functional with the gradient corrected Lee, Yang, and Parr (LYP) ${ }^{51}$ correlation functional, whereas the hybrid B3LYP functional contains the three parameter exchange of Becke (B3) ${ }^{52}$ with the LYP correlation functional. All calculations were performed using tight convergence criteria. ${ }^{53}$ Analysis of the atomic charges and spin densities was performed using the natural population analysis (NPA) framework developed by Weinhold et al. ${ }^{54}$ Calculations were carried out using the Michigan State University Chemistry Supercomputer Facility.

Geometry Optimizations. The initial geometries of the molecules were generated using SPARTAN ${ }^{55}$ or GaussView ${ }^{56}$ and subsequently optimized in three steps. The first optimization was performed using restricted open-shell Hartree-Fock (ROHF) theory with the STO-3G** basis set. These structures were then subjected to a second optimization using the restricted openshell density functional ROBLYP with the 3-21G** basis set, followed by a third optimization using the ROB3LYP functional with the $6-31 \mathrm{G}^{*}$ basis set. Frequency calculations at the ROB3LYP/6-31G* level of theory were performed on the final optimized structures to ensure that these geometries corresponded to global minima. In several cases $\left(\mathrm{R}=\mathrm{NH}_{2}, \mathrm{NMe}_{2}\right.$, $\mathrm{OMe}$, and $\mathrm{OEt}$ ), imaginary frequencies were obtained. For these systems, the molecules were distorted along those coordinates in order to find the lowest energy structure. The structure was then reoptimized at the ROB3LYP/6-31G* level and subjected to a subsequent frequency calculation. This procedure was repeated until no imaginary frequencies were found. In all cases, the final optimized structures were used in the single point calculations described below. 
Single Point Calculations. Single point calculations were performed using the unrestricted density functional UB3LYP with the $6-311 \mathrm{G}^{* *}$ basis set assuming a doublet ground state and a molecular charge of zero. The maximum error for the expectation value of the spin operator $\left\langle\mathbf{S}^{2}\right\rangle$ differed from the theoretical value for a pure spin state of $S(S+1)$ (where $S$ is the total spin) by an average error of $4.3 \%$, and in no case exceeded $6 \%$, indicating the degree of spin contamination in the Kohn-Sham Slater determinant is negligible for the molecules investigated. ${ }^{15,57,58}$ The UBLYP functional with the 6-311G** basis was also used on a subset of substituents $(\mathrm{H}$, $\mathrm{Cl}, \mathrm{OMe}, \mathrm{NH}_{2}, \mathrm{NO}_{2}$, and $\mathrm{CF}_{3}$ ) to determine whether any calculated trends in charge and/or spin density distribution were functional dependent. Although specific values for charge and spin density were different, trends in structure, spin and charge density across the series were qualitatively similar. To simplify the discussion, the results reported here derive from calculations at the UB3LYP/6-311G** level.

The substituents examined in these calculations were selected to cover a wide range of possible interactions with the phenoxyl ring and included $\sigma$-donors (Me, $t$-butyl, and TMS), a $\sigma$-acceptor $\left(\mathrm{CF}_{3}\right), \pi$-donors $\left(\mathrm{OH}, \mathrm{OMe}, \mathrm{OEt}, \mathrm{NH}_{2}\right.$, and $\left.\mathrm{NMe}_{2}\right), \pi$-acceptors $\left(\mathrm{NO}_{2}\right.$ and $\left.\mathrm{CN}\right), \pi$-donor/ $\sigma$-acceptors $(\mathrm{F}, \mathrm{Cl}$, and $\mathrm{Br})$, and a $\pi$-acceptor $/ \sigma$-donor (vinyl). In all cases the unsubstituted phenoxy radical (i.e., $\mathrm{R}=\mathrm{H}$ ) was used as a reference point for comparisons.

\section{Results and Discussion}

Geometries of para-Substituted Phenoxy Radicals. A crystal structure for the phenoxy radical has not been reported, however, it has been studied extensively using a wide range of computational methods. ${ }^{43,44}$ Based on comparisons to the unsubstituted compound, our results indicate that the geometry of the phenoxy radical in terms of both bond distances and bond angles is largely insensitive to changes in the para substituent on the ring. The most significant perturbation arises in the $\mathrm{C}-\mathrm{O}$ distance for the vinyl derivative, where a distance of $1.247 \AA$ (versus $1.257 \AA$ for $\mathrm{R}=\mathrm{H}$ ) was noted. A slight increase in double bond character was also observed for the $\mathrm{NO}_{2-}$ and $\mathrm{CN}$ substituted compounds, though to a lesser extent. The resonance structures in Scheme 1 illustrate that the origin of double-bond character in the $\mathrm{C}-\mathrm{O}$ bond arises from delocalization of lonepair electrons on the oxygen into the ring. The slight increases in bond order observed for the three $\pi$-accepting substituents in this study can therefore be viewed as a reflection of this resonance effect. The influence of electron delocalization will be expanded upon later in the discussion in the context of spin polarization, but in terms of geometry, this appears to be the only significant structural perturbation across this series.

Spin and Charge Density Movement in para-Substituted Phenoxy Radicals. A. Overview. Our work was originally motivated by an interest in magnetic exchange interactions between transition metals and bound organic radicals such as orthosemiquinones. ${ }^{59-61}$ For such systems, significant negative charge density at the oxygen atoms coupled with varying degrees of unpaired spin density would provide an entry into a series of compounds having tunable magnetic properties. Our initial computational efforts on this class of compounds were inconclusive, most likely due to the presence of competing effects from substituents that simultaneously have different regiospecific interactions with the two ligating oxygen atoms. We therefore sought to simplify the problem sufficient to extract the basic phenomenological effects of compositional changes on spin and

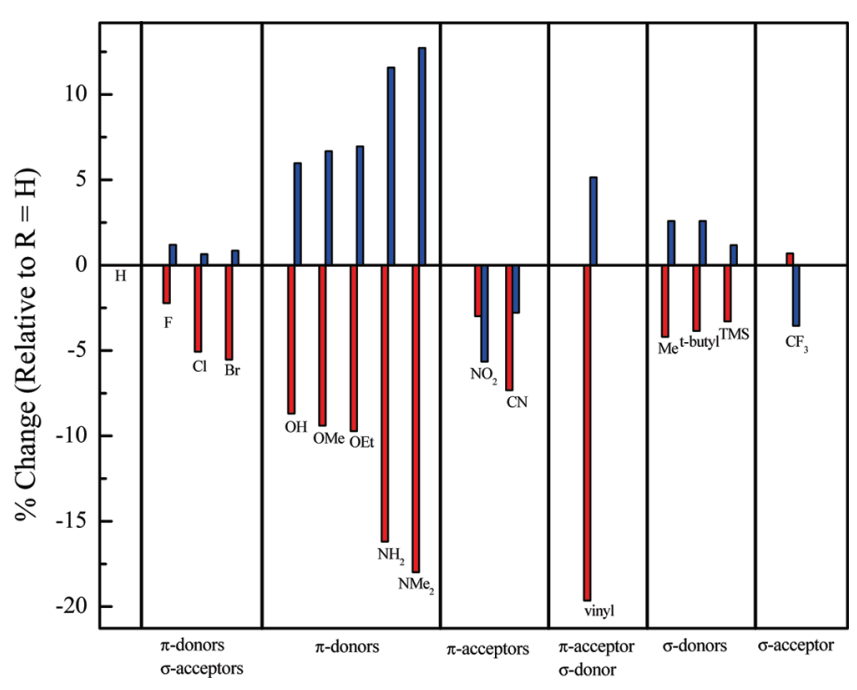

Figure 1. Shifts in charge (blue) and unpaired spin (red) density at the oxygen atom for a series of para-substituted phenoxy radicals. The $\%$ change in both parameters is referenced to values obtained for the unsubstituted compound (i.e., $\mathrm{R}=\mathrm{H}$, for which spin $=0.439$ $\alpha$-electrons and charge $=-0.484$ electrons). A positive change indicates an increase in the magnitude of negative charge density or an increase in spin density.

charge polarization that we could then use as a guide for analyses of more complex systems.

A graphical depiction of our computational results is shown in Figure 1. Consistent with previous reports ${ }^{44}$ this plot clearly illustrates that differential polarization of spin and charge is possible, even facile, given the proper choice of substituent. In fact, there are far more examples evident where shifts in spin and charge density oppose rather than track each other. In principle, there are four regimes of spin and charge polarization that could be sampled corresponding to an increase and/or decrease of spin and/or charge at the oxygen atom. Figure 1 illustrates that three of these four regimes were realized; none of the substituents we chose gave rise to a simultaneous increase in both spin and charge density at the oxygen atom. The degree of polarization varies considerably across the series although the most significant effects appear to be associated with substituents that can couple strongly to the $\pi$-system of the molecule. The magnitude of the effect in some cases is substantial: dimethylamine, for example, causes a nearly $20 \%$ reduction in spin density at the oxygen atom as compared to the unsubstituted phenoxy radical, while at the same time serves to increase the negative charge density at oxygen by over $10 \%$. The vinyl group appears to induce a similar effect on spin, though its influence on charge density is less pronounced. Even seemingly innocuous substituents such as methyl and $t$-butyl are predicted to differentially polarize spin and charge. It is interesting to note that, in general, the substituent effect on spin polarization appears to be larger in a relative sense than the corresponding shift in charge, though exceptions to this trend are apparent (e.g., $\mathrm{R}=\mathrm{NO}_{2}$ ).

Clearly, spin and charge are responding differently to the perturbations introduced by the various substituents. In order for this to be of use with regard to molecular design, however, the underlying mechanism(s) giving rise to these observations must be understood.

B. Substituent Effects on Charge Density Distribution. Computational methods have been employed by many different groups in an effort to describe charge distributions in molecules. ${ }^{62-64}$ Of greatest relevance to our work is the 


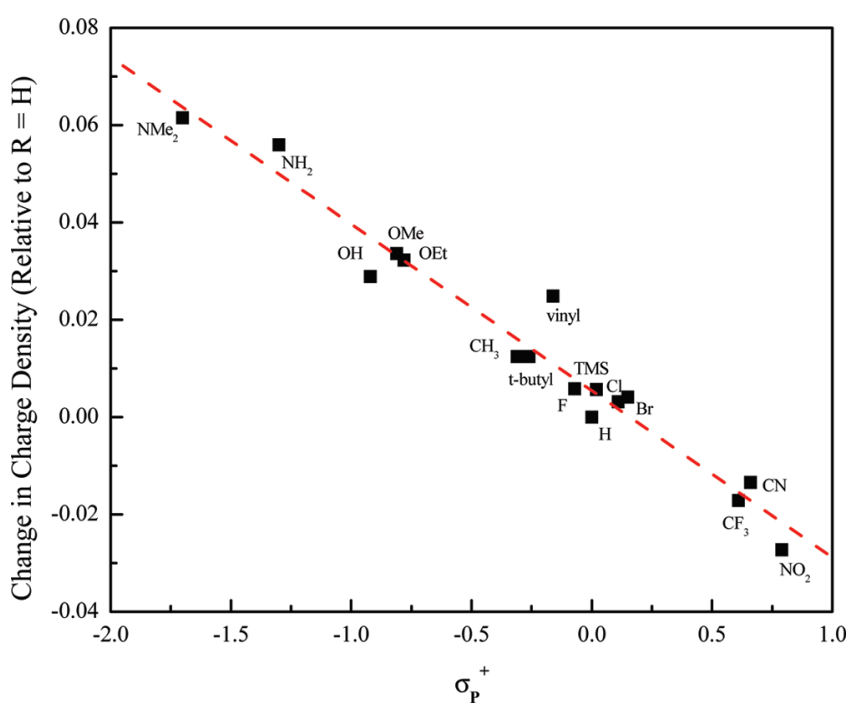

Figure 2. Plot of the calculated change in charge density at the oxygen atom as a function of the $\sigma_{\mathrm{p}}^{+}$coefficient of the R-group in parasubstituted phenoxy radicals. The value given is relative to that obtained for the unsubstituted radical (i.e., $\mathrm{R}=\mathrm{H}$ ); a positive number indicates an increase in negative charge density at the oxygen atom. The $\sigma_{\mathrm{p}}{ }^{+}$ values were taken from the review by Hansch, Leo, and Taft (cf. 4). See text for further details.

aforementioned study by $\mathrm{Wu}$ and Lai, who employed density functional theory to examine the effect of para substituents on charge density polarization in phenoxy radicals. ${ }^{44}$ These authors demonstrated that the change in charge density at the oxygen atom correlates well with the Hammett-Brown ${ }^{65}$ polar substituent constant $\left(\sigma_{\mathrm{p}}^{+}\right)$. The $\sigma_{\mathrm{p}}^{+}$constants derive from data on the solvolysis of $t$-cumyl chlorides, an $\mathrm{S}_{\mathrm{N}} 1$ reaction whose rate depends on the electrophilicity of the tertiary carbocation associated with the transition state. Thus, in the most common interpretation, ${ }^{66}$ the $\sigma_{\mathrm{p}}{ }^{+}$constant reflects the ability of a substituent to donate or remove charge density; based on its original definition, ${ }^{65}$ negative values of ${\sigma_{\mathrm{p}}}^{+}$correspond to charge-donating substituents whereas positive values are considered electron-withdrawing (all relative to hydrogen). The $\sigma_{\mathrm{p}}{ }^{+}$ constants broadly encompass both inductive ( $\sigma$-donating or $\sigma$-accepting) and resonance ( $\pi$-accepting or $\pi$-donating) effects associated with the substituent.

The results of our calculations fully support the work of Wu and Lai; a plot of the change in charge density at the oxygen atom as a function of the $\sigma_{\mathrm{p}}{ }^{+}$coefficient for each substituent that we examined is shown in Figure 2. The correlation in Figure 2 indicates that the individual resonance and inductive effects of the substituents are effective in modulating the charge density at the oxygen atom, with electron-donating and withdrawing substituents increasing and decreasing, respectively, the negative charge density at the oxygen atom. Overall, the results in Figure 2 conform to our qualitative expectations given the electronegativity of oxygen and the tendency of $\pi$-donors to increase electron density within an aromatic ring system. On a more quantitative level, the linearity of the correlation is consistent with what one expects from a Hammett-type plot, though the underlying reason for the empirical success of such plots is the subject of continued debate. ${ }^{67}$ Experimental evidence indicating a correlation between ${\sigma_{\mathrm{p}}}^{+}$and the reduction potentials for phenoxy radicals (i.e., $\mathrm{PhO}^{\bullet}+\mathrm{e}^{-} \rightarrow \mathrm{PhO}^{-}$) supports the notion that electron deficient radicals are stabilized by the transfer of negative charge density from the substituent to the oxygen radical. ${ }^{6}$ The correlation in Figure 2 coupled with the linear free energy relationship upon which the $\sigma_{\mathrm{p}}{ }^{+}$constants are based can therefore be interpreted as reflecting the ability of the substituent to inductively and/or resonantly stabilize the oxygen radical via the modulation of charge density within the molecule. ${ }^{67}$

C. Substituent Effects on Spin Polarization. The ability of substituents to influence the distribution of spin density within an aromatic system can be understood qualitatively by extending the resonance picture for the phenoxy radical as shown in Scheme 3 . In the case of $\pi$-donors (A), adherence to the Pauli principle requires that the interaction between the lone-pair of the donor and the aromatic ring involve the effective transfer of an electron whose polarization is opposite to that associated with the carbon at the para position: arbitrarily assigning the spin density within the ring as $\alpha$, substituents with lone pairs having $\pi$-symmetry can therefore be viewed as $\beta$-spin donors. The consequence of this interaction is the creation of residual $\alpha$-spin on the $\mathrm{R}$ group and a net redistribution of spin density out of the aromatic ring. ${ }^{69}$ Taken to the extreme, complete transfer of the electron from the donor gives rise to the zwitterion depicted on the right side of Scheme 3A, a description reminiscent of the three-electron bonding schemes often invoked to describe such systems. ${ }^{70}$ Groups that can act as $\pi$-acceptors (e.g., vinyl) can be described in a similar manner (B). Their ability to conjugate to the ring lends to their depiction as $\alpha$-spin acceptors, however, the net effect in terms of the redistribution of spin polarization to the periphery of the substituent is largely the same as seen for $\pi$-donors.

The mechanism by which $\sigma$-donors/acceptors influence spin polarization is not as straightforward but can still be viewed in terms of a direct orbital interaction between the substituent and the radical-containing $\pi$-system of the ring through hyperconjugation (C). In this context, aliphatic groups such as $\mathrm{CH}_{3}$ can be considered $\beta$-spin donors wherein a $\sigma$ orbital of the $\mathrm{C}-\mathrm{H}$ fragment plays a role similar to that depicted for the $\pi$-donors. An important difference is that the net transfer of $\beta$-spin density from the $\mathrm{C}-\mathrm{H}$ fragment to the para-carbon will serve to destabilize the bonding within the substituent by virtue of delocalization of the bonding pair of electrons to the phenoxy $\pi$ orbital: this could explain, in part, the generally smaller polarization effect observed for the $\sigma$-donor/acceptor-substituents relative to their $\pi$-based counterparts.

Common to all three of the spin polarization mechanisms illustrated in Scheme 3 is the ability of the substituent to couple into the $\pi$ system to redistribute spin density out of the aromatic ring. Although useful from a qualitative perspective, the scenarios presented above still do not provide sufficient insight as to the relative effectiveness of one substituent versus another at polarizing spin density, nor do they allow us to understand why spin and charge behave independently in terms of the directionality of their polarizations.

Previous Studies. A number of groups have considered substituent effects on spin polarization by correlating spin density distribution with various physical properties (both calculated and experimental). Both $\mathrm{Wu}$ and Lai ${ }^{44}$ and Brinck et al. ${ }^{71}$ have examined the link between spin density polarization and thermodynamics by calculating the $\mathrm{O}-\mathrm{H}$ bond dissociation energy (BDE) of substituted phenols. A correlation between decreasing $\mathrm{BDE}$ and the presence of $\pi$-donors was noted, suggesting that stabilization of the radical is associated with increased spin delocalization of the odd electron. ${ }^{71}$ The influence of para substituents on the phenoxy radical has also been investigated in an AM1 study by Bean, ${ }^{72}$ who likewise focused on bond dissociation energies of substituted phenols. Bean concluded that the relative stability of the radicals could be 
SCHEME 3: Spin Delocalization Mechanisms in Phenoxy Radicals: (A) $\beta$-Spin Donor ( $\pi$-Donors); (B) Conjugative $\alpha$-Spin Acceptor ( $\pi$-Acceptors); (C) Hyperconjugative $\beta$-Spin Donors ( $\sigma$-Donors)<smiles>O=C1C=CC(=O)C(C2CCCCC2[18O])=C1</smiles>

A<smiles>[R7]C=C1C=CC(=O)C=C1</smiles>

B<smiles></smiles><smiles>C1#CC#C1</smiles><smiles>CC(C)(O)OC1C=CC(=O)C=C1</smiles>

C

understood in the context of the captodative effect, ${ }^{73-76}$ a concept first introduced by Dewar ${ }^{77}$ in which a combination of donors and acceptors within the same compound led to a "push-pull" effect that ultimately stabilized the radical. This model holds that a strong electron-donating group in the para position of the ring leads to stabilization of the radical (conversely, an electron-withdrawing group would destabilize the radical via an anticaptodative effect). The validity of this idea garners support from the results of $\mathrm{Wu}$ and Lai and Brinck et al., ${ }^{44,71}$ and to some extent our own, insofar as strong donors are observed to promote redistribution of spin polarization away from the oxygen atom and toward the substituent.

Correlations with bond dissociation energies represent one of many ways in which workers have sought to systematize the effects of substituents on spin polarization. These efforts have resulted in numerous Hammett-type radical scales: among the more commonly employed are (1) the $\sigma_{\mathrm{JJ}}{ }^{\circ}$ scale of Jiang and $\mathrm{Ji},{ }^{80,81}$ which is based on cycloaddition reactions of $\alpha, \beta, \beta-$ trifluorostyrenes, (2) the $\sigma_{\alpha}{ }^{\circ}$ scale of Arnold, ${ }^{69,80}$ which relates spin density to hyperfine interactions in substituted benzyl radicals, (3) $\sigma_{\mathrm{C}}^{*}$, which Creary used to describe the rearrangement of 2-aryl-3,3-dimethylmethylenecyclopropanes, ${ }^{81,82}$ (4)
Jackson's $\sigma_{\mathrm{J}}{ }^{*}$ parameter, which was derived from the thermal decomposition of dibenzyl mercurials, ${ }^{83,84}(5)$ Fisher's $\sigma_{\mathrm{F}}{ }^{\circ}$ parameter based on the bromination of 4-substituted 3-cyanotoluenes by NBS, ${ }^{85,86}$ and (6) the $\Delta \mathrm{D}$ scale of Adam, ${ }^{65}$ which correlates spin density with zero-field splitting in substituted 1,3-diarylcyclopentane triplet diradicals. The success of these various parameters at describing substituent effects on spin polarization in a broader context has been mixed. For example, $\mathrm{Wu}$ and Lai modeled the change in spin density $(\Delta S)$ at the oxygen atom in their study using $\sigma_{\mathrm{JJ}}{ }^{\circ}$; the authors acknowledged that the correlation they reported was not as compelling as what they observed between $\sigma_{\mathrm{p}}{ }^{+}$and charge density, although it was clear that spin delocalization was maximized with $\pi$-donor ligands. It should be noted that these authors made a point of noting that spin delocalization effects in the phenoxy system could be quite different than in the benzyl radicals upon which the $\sigma_{\mathrm{JJ}}{ }^{\circ}$ constants are based, implying that a correlation between spin density and $\sigma_{\mathrm{JJ}}{ }^{\circ}$ perhaps should not be expected in the case of substituted phenoxy radicals.

In Figure 3 are shown our calculated results for the 15 substituents examined in this study plotted against the four Hammett parameters most commonly invoked to describe 
A.

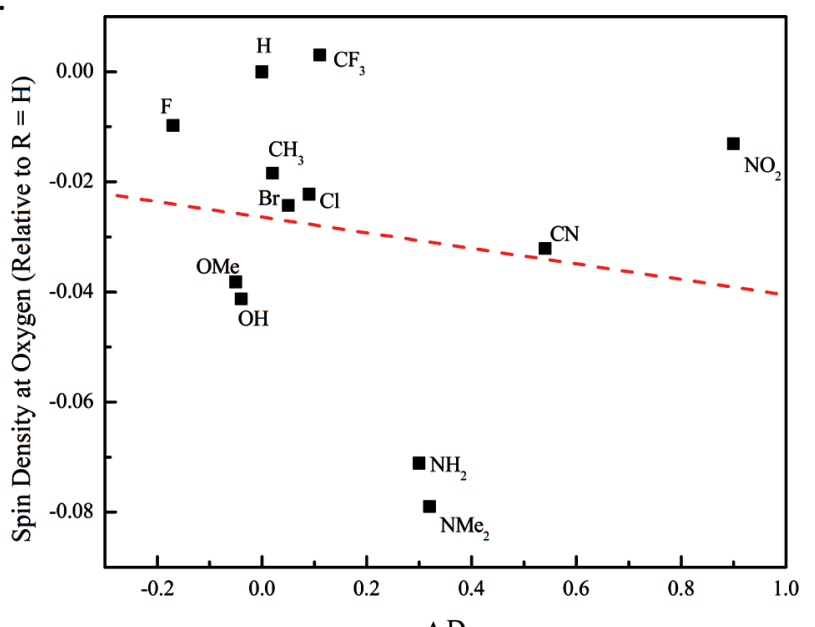

C.

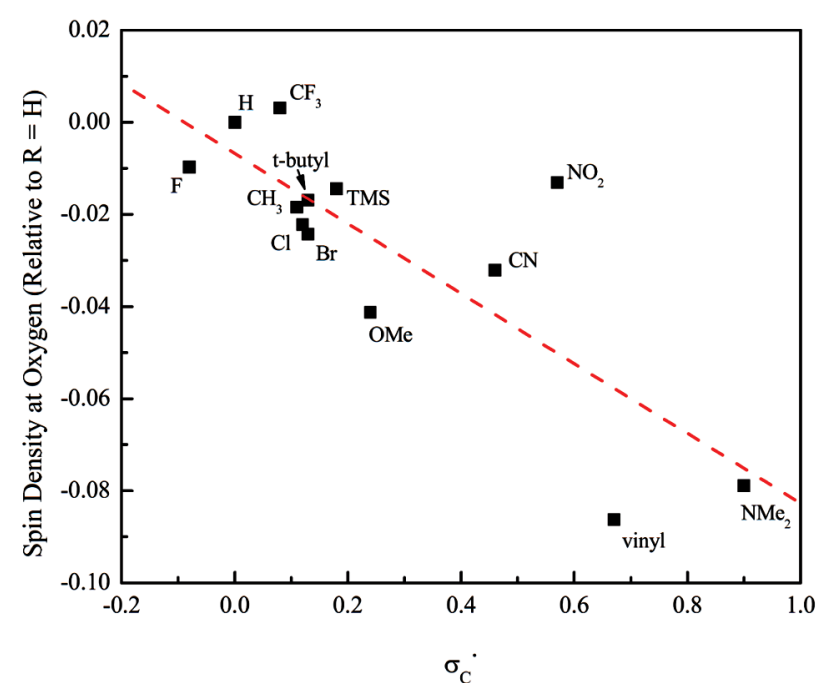

B.
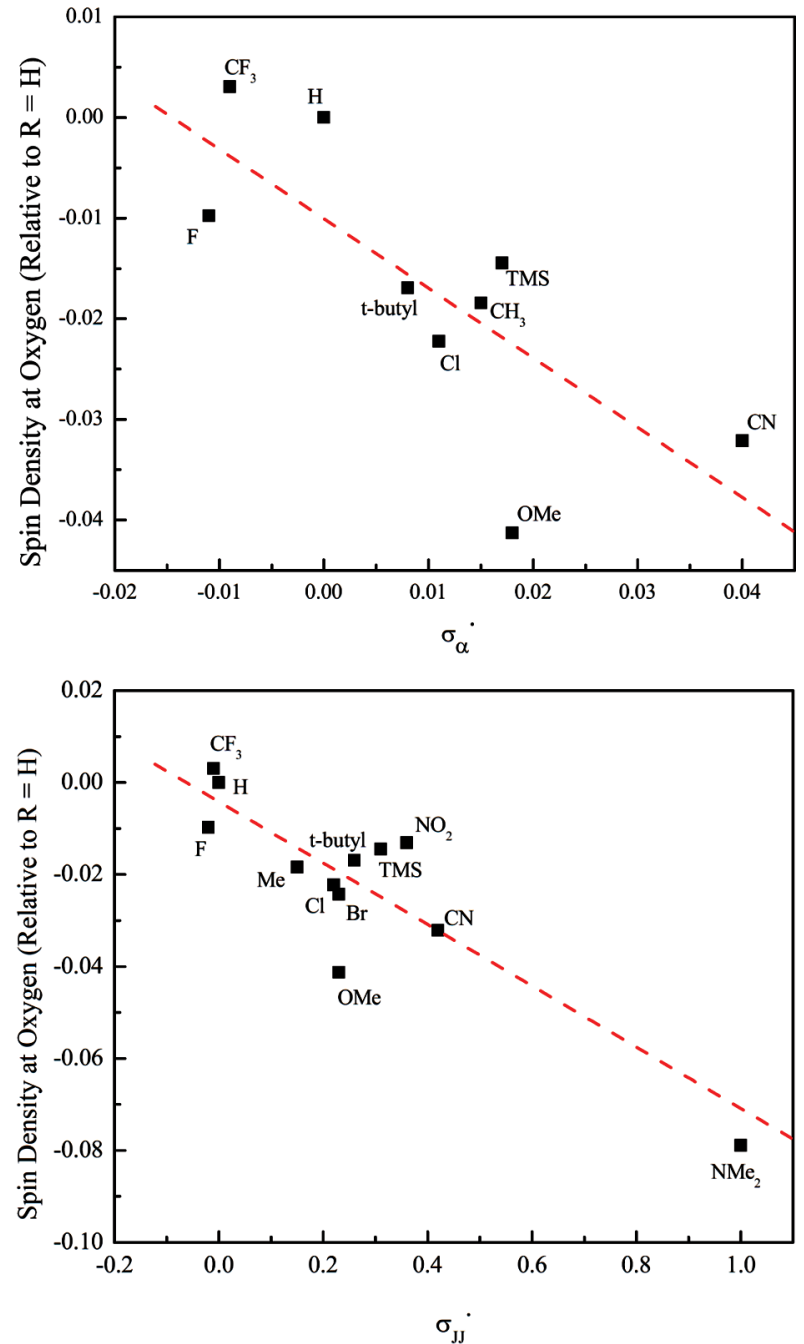

Figure 3. Plots of the change in spin density at the oxygen atom of para-substituted phenoxy radicals as a function of the $\Delta \mathrm{D}(\mathrm{A}), \sigma_{\alpha}{ }^{\circ}(\mathrm{B}), \sigma_{\mathrm{C}}{ }^{\circ}$ (C), and $\sigma_{\mathrm{JJ}}{ }^{\circ}$ (D) Hammett-type spin delocalization parameters.

substituent effects on spin distribution. It can be seen upon inspection that none of these parameters yields a particularly good correlation. Indeed, the $\Delta \mathrm{D}$ parameter, which we initially believed held the greatest promise due to the fact that zerofield splitting in diradicals should correlate quite well with unpaired spin density, actually does the worst in terms of describing the polarization of spin away from the oxygen atom in the phenoxy radical. The correlation appears to be strongest with the $\sigma_{\mathrm{JJ}}{ }^{\circ}$ parameter, similar to what was reported by $\mathrm{Wu}$ and Lai, though in our case this observation is clearly dependent upon inclusion of the value for the dimethylamino derivative. Figure 3 reinforces the suggestion by $\mathrm{Wu}$ and Lai that while these various spin delocalization scales may work quite well for their parent systems they do not appear to be transferable between different systems to any significant degree. ${ }^{71} \mathrm{We}$ therefore looked to our calculations to see if there was another, more robust marker for spin polarization that could provide us with greater insight into the origin of these substituent effects.

HOMO-LUMO Gap Correlation. After an extensive analysis of our data, we uncovered an empirical relationship between the polarization of spin density and the energy gap between the highest-occupied and lowest-unoccupied molecular orbitals of the molecules. Before we describe these results, we must first define what we mean by the LUMO of a molecule within the context of this study.
As mentioned in the Experimental section, we carried out our calculations using a spin-unrestricted formalism. This approach is necessary in order to allow the spatial characteristics of each spin polarization to behave independently, but a drawback of this method is that the orbitals produced within this formalism are one-electron constructs. As a result, each oneelectron orbital of the molecule is a distinct eigenvector with its own eigenvalue. The challenge is therefore to correlate these one-electron orbitals with a two-electron orbital picture of the kind that chemists typically invoke to describe bonding interactions in molecules. Amos and Hall have argued that this can be done in a straightforward manner provided that the spatial overlap of a given pair of $\alpha$ - and $\beta$-spin orbitals approaches unity ${ }^{87-89}$ In this circumstance, the $\alpha$ - and $\beta$-spin one-electron orbitals calculated within the spin-unrestricted formalism can be thought of as describing essentially the same molecular orbital (i.e., the corresponding two-electron orbital), differing only in the polarization of the electron.

This idea can be illustrated by examining the relevant wavefunctions of one of the compounds from our study. Energetically, the true HOMO-LUMO gap in an $\mathrm{S}=1 / 2$ system within a spin-unrestricted formalism is typically the $\alpha$-HOMO$\beta$-LUMO gap: this was found to be the case in all of the molecules we examined. In Figure 4 are shown the $\alpha$-HOMO, $\beta$-LUMO, and $\alpha$-LUMO of the $p$ - $\mathrm{NH}_{2}$-phenoxyl radical. It can 


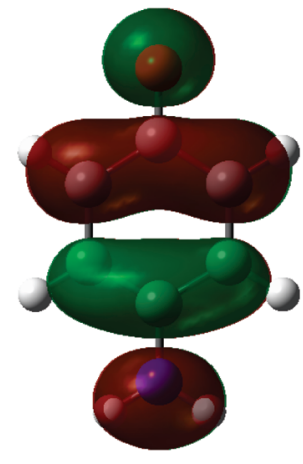

$\alpha-H O M O$

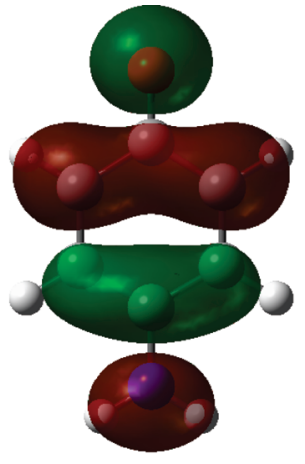

B-LUMO

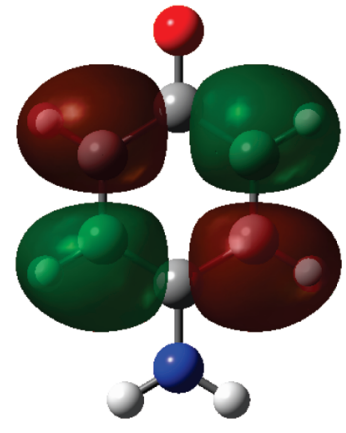

$\alpha-L U M O$

Figure 4. Comparison of the $\alpha$-HOMO, $\beta$-LUMO, and $\alpha$-LUMO Kohn-Sham molecular orbitals of the $p$ - $\mathrm{NH}_{2}$-phenoxy radical. The orbitals were generated at the UB3LYP/6-311G** level of theory and visualized using GaussView with an isosurface value of 0.020 .

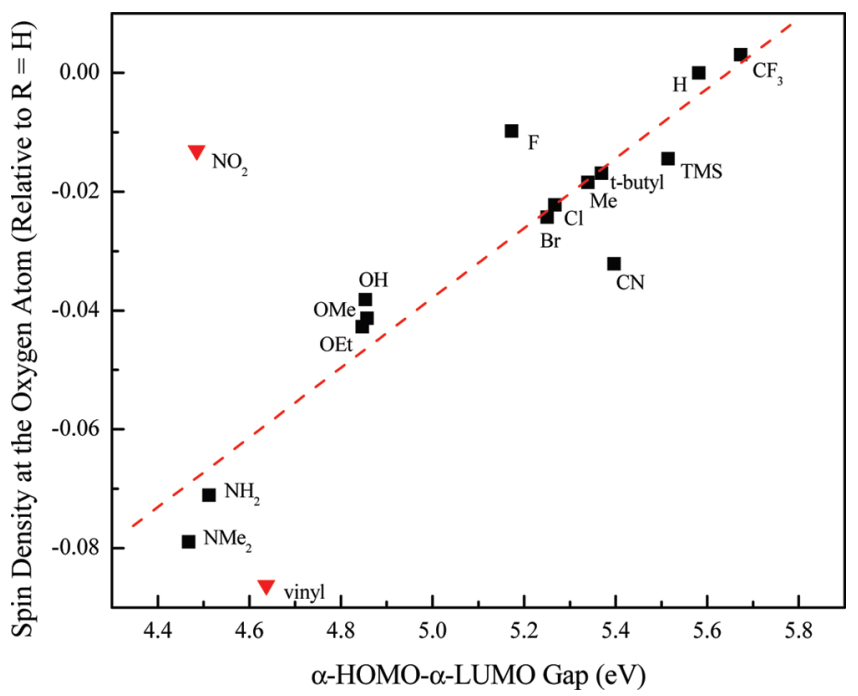

Figure 5. Plot of the calculated changes in spin density at the oxygen atom versus the $\alpha$-HOMO- $\alpha$-LUMO gaps for a series of parasubstituted phenoxy radicals calculated at the UB3LYP/6-311G** level of theory.

be seen that the $\alpha$-HOMO and $\beta$-LUMO orbitals are essentially identical in terms of their spatial properties, ${ }^{90}$ whereas the $\alpha$-LUMO possesses completely distinct bonding characteristics. Within the context of Amos and Hall's corresponding orbital transformation, ${ }^{87-89}$ the $\alpha$-HOMO and $\beta$-LUMO, despite having different eigenvalues in the spin-unrestricted calculation, can be viewed as representing the same molecular orbital when projected onto a two-electron picture. An analogous result was found for all 15 compounds examined in this study. We therefore believe that the $\alpha$-LUMO represents the physically significant orbital for defining the HOMO-LUMO gaps of these open-shell systems. ${ }^{91-95}$

A plot of the change in spin density at the oxygen atom versus the HOMO-LUMO gap of all 15 of the para-substituted phenoxy radicals we examined is shown in Figure 5. Our results clearly show a strong correlation between these two variables. A comparison of these results with Figure 3 further illustrates that the HOMO-LUMO gap is a significantly better marker for changes in spin density across this series than any of the commonly invoked Hammett-type radical constants. ${ }^{96}$ It is worth noting that qualitatively similar results were found at the UHF/ $6-311 \mathrm{G}^{* *}$ level of theory, suggesting that this correlation is insensitive to the theoretical method employed. Empirically, we observe that molecules with smaller HOMO-LUMO gaps exhibit a greater degree of spin polarization away from the oxygen atom: closer inspection reveals that this region is dominated by $\pi$-donating substituents, whereas larger HOMOLUMO gaps are associated with substituents whose primary influence is $\sigma$-based. It is interesting to note that, although the general grouping of substituents (e.g., $\pi$-donors, $\sigma$-acceptors, etc.) is similar to what was observed for charge polarization effects, the specific ordering within these subgroups is different. The correlation is clearly not perfect. In particular, the $\mathrm{NO}_{2}$ and vinyl derivatives fall significantly off the line, with the former having a smaller gap than expected whereas $p$-vinyl-phenoxyl exhibits an anomalously large splitting. Nevertheless, the overall trend evident in Figure 5 suggests that the factors influencing spin polarization and the HOMO-LUMO gap in this series of compounds are related.

D. Mechanism of Spin Polarization. The correlation revealed by Figure 5 can be explained in the context of the simple molecular orbital scheme shown in Figure 6. In accord with the resonance picture illustrated in Scheme 3, the dominant interaction is proposed to occur between the lone pair associated with the $\pi$-donor and the $\pi$-orbital(s) containing the unpaired electron of the phenoxy radical. ${ }^{70,97}$ Assuming that the degree of spatial overlap between the donor and the $\pi$-system of the ring is roughly invariant, the extent of destabilization of the HOMO will be dictated primarily by the relative energies of the $\phi_{\pi \text {-donor }}$ and $\phi_{\text {phenoxy-somo orbitals: as these two orbitals }}$ become more energetically equivalent the degree of this destabilization will increase. This will have two immediate consequences: (1) The wavefunction corresponding to the SOMO of the adduct will exhibit an enhanced contribution from the substituent, the net effect of which will be to redistribute the electron density associated with the unpaired electron onto the substituent and away from the oxygen atom; and (2) the HOMO-LUMO gap will decrease provided the $\alpha$-LUMO of the phenoxy radical is essentially nonbonding with respect to the substituent.

Details from the calculations we have carried out provide considerable support for this picture. For example, it can be seen in Figure 5 that decreasing the electronegativity of the donor atom from oxygen in $\mathrm{R}=\mathrm{OMe}$ to nitrogen in $\mathrm{R}=\mathrm{NMe}_{2}$ leads to a smaller HOMO-LUMO gap, consistent with the notion that the lone pair of nitrogen in dimethylamine should present a better energetic match with the aromatic ring than does the methoxy group. More compelling is the fact that the redistribution of electron density due to mixing between the $\phi_{\text {phenoxy-SOMo }}$ and $\phi_{\pi \text {-donor }}$ orbitals suggested in Figure 6 is reflected in the spatial characteristics of the wavefunction. The orbital 

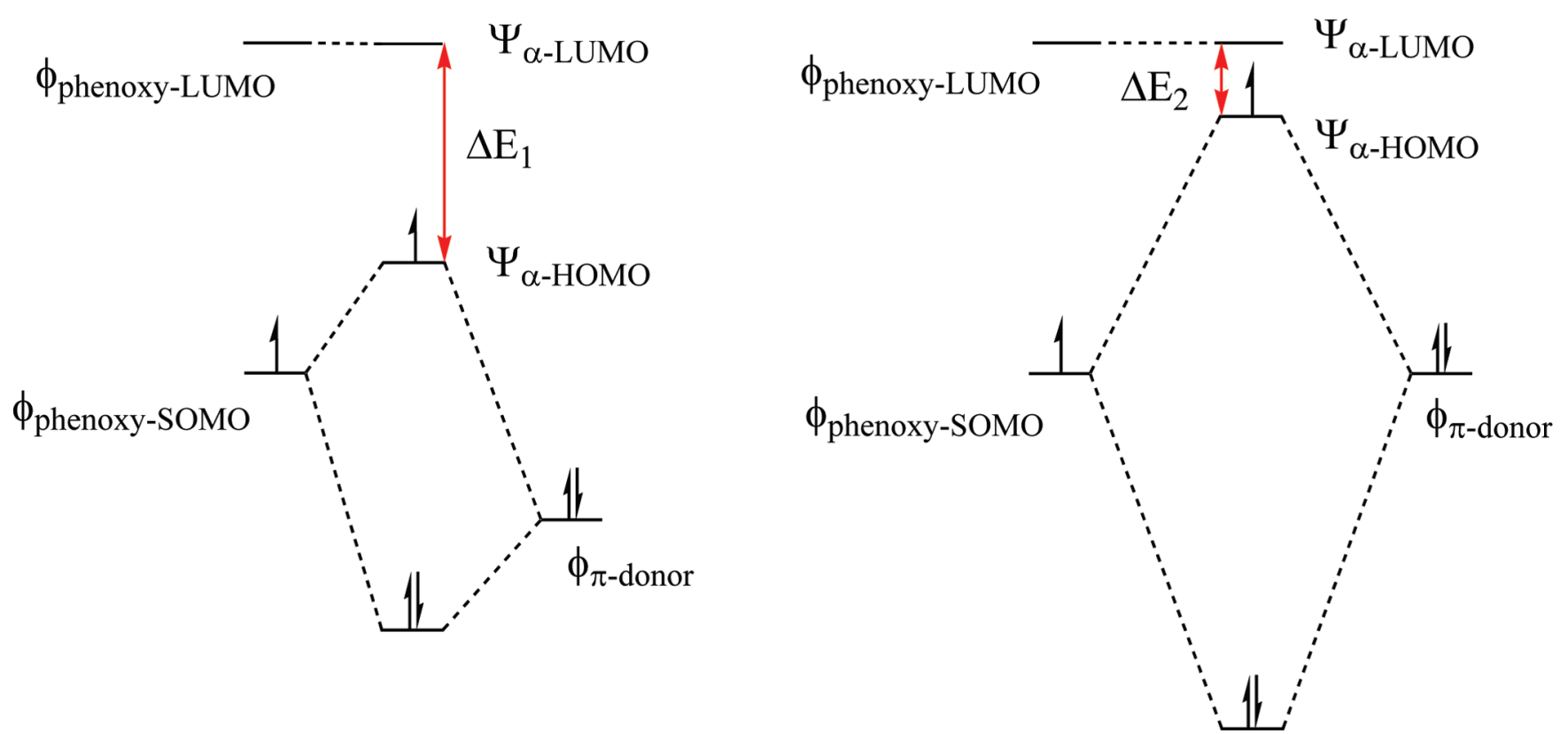

Figure 6. Molecular orbital-based description of the spin delocalization mechanism proposed for $\pi$-donor-containing phenoxy radicals. The interaction of the $\phi_{\pi \text {-donor }}$ orbital of the substituent with the phenoxy $\phi_{\text {phenoxy-somo }}$ results in a net one-electron stabilization. The degree of interaction between these two orbitals dictates the $\alpha$-HOMO- $\alpha$-LUMO energy gap, which in turn alters the composition of the $\alpha$-HOMO and thus the spatial distribution of unpaired spin density.

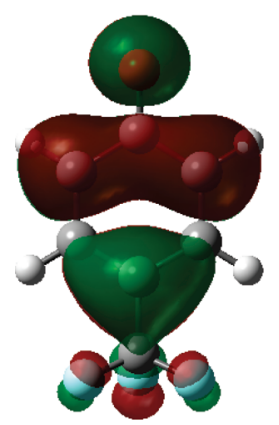

p- $\mathrm{CF}_{3}$-phenoxyl

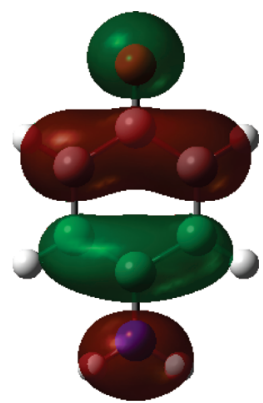

p- $\mathrm{NH}_{2}$-phenoxyl

Figure 7. Comparison between the Kohn-Sham $\alpha$-HOMO molecular orbitals of $p-\mathrm{CF}_{3}$ - and $p-\mathrm{NH}_{2}$-substituted phenoxy radicals.

housing the unpaired electron in these compounds can be approximately defined as

$$
\Psi_{\alpha-\mathrm{HOMO}}=c_{1} \phi_{\text {phenoxy-SOMO }}+c_{2} \phi_{\pi-\text { donor }}
$$

where $c_{1}^{2}+c_{2}^{2}=1$. As the $\phi_{\pi \text {-donor }}$ orbital increases in energy (i.e., shifting from left to right in Figure 6), more extensive orbital mixing should result in an increase in $c_{2}$ at the expense of $c_{1}$. The data in Table 1 confirm these expectations: as the HOMO-LUMO gap decreases, the wavefunction corresponding to the HOMO exhibits a systematic increase in the percent contribution from the substituent $(\% \mathrm{R})$ and a corresponding decrease in oxygen character $(\% \mathrm{O})$. Since the unpaired electron carries all of the excess spin density of the molecule, the delocalization of the SOMO onto the substituent implied by its increased contribution to the wavefunction has the net effect of polarizing spin away from the oxygen atom. This is illustrated in Figure 7 , in which the $\pi$ - and $\sigma$-donating properties of the $\mathrm{NH}_{2}$ and $\mathrm{CF}_{3}$ groups, respectively, have dramatically different effects on the spatial properties of the $\alpha$-HOMO. This simple MO explanation, which essentially expands upon the concept

of the captodative effect ${ }^{74,76}$ and three-electron bonding, ${ }^{70,98}$ thus accounts in a general way for the correlation evident in Figure 5.

Deviations from the HOMO-LUMO Gap Correlation. Implicit in the model just described is the notion that the $\Psi_{\alpha \text {-LUMO }}$ is not perturbed upon incorporation of the substituent. Defining the $\alpha$-LUMO as

$$
\Psi_{\alpha-\text { LUMO }}=c_{3} \phi_{\text {phenoxy-LUMO }}+c_{4} \phi_{\pi^{*} \text {-substituent }}
$$

we can see from Table 1 that $c_{4}$ is, in fact, either zero or relatively small (i.e., $<10 \%$ ) for most of the substituents included in our study. The two glaring exceptions to this are $\mathrm{NO}_{2}$ and vinyl: these groups contribute $69 \%$ and $48 \%$ to their respective LUMOs. We now consider each of these cases to determine whether their properties can be understood within the context of the model we have developed.

The results we obtained for $p$ - $\mathrm{NO}_{2}$-phenoxyl constitute the most significant deviation from the HOMO-LUMO gap correlation illustrated in Figure 5. Specifically, our calculations indicate that the HOMO-LUMO gap for this compound is substantially smaller than expected based on the calculated redistribution of spin density away from the oxygen atom. Figure 8 presents a comparison of the LUMOs for phenoxyl and $p-\mathrm{NH}_{2}-$ phenoxyl with the LUMO and LUMO+1 of $p-\mathrm{NO}_{2}$-phenoxyl. These plots clearly show that the character of the LUMO is different for $p-\mathrm{NO}_{2}$-phenoxyl: the orbital corresponding to the LUMO in most of the other compounds in our study is now the next-highest energy orbital (LUMO+1) in $p-\mathrm{NO}_{2}$-phenoxyl. This situation arises due to the presence of a low-lying $\pi^{*}$ acceptor orbital associated with the $\mathrm{NO}_{2}$ group, allowing for a new interaction between the substituent and the LUMO+1 of the free phenoxyl moiety and the stabilization of that orbital in $p$ - $\mathrm{NO}_{2}$-phenoxyl. A qualitative molecular orbital diagram depicting this situation is shown in Figure 9. It should be noted that the polarization of spin density is still driven by the character of the HOMO as described above: Table 1 shows that 
TABLE 1: Percent Atomic Contributions for Oxygen $(\% O)^{a}$ and the Substituent $(\% \text { R })^{a}$ Group in the $\alpha-H O M O$ and $\alpha-L U M O$ Kohn-Sham MO's for a Series of para-Phenoxy Radicals

\begin{tabular}{|c|c|c|c|c|c|}
\hline $\mathrm{R}$ & $\% \mathrm{O} \alpha-\mathrm{HOMO}$ & $\% \mathrm{R} \alpha-\mathrm{HOMO}$ & $\% \mathrm{O} \alpha-\mathrm{LUMO}$ & $\% \mathrm{R} \alpha-\mathrm{LUMO}$ & $\Delta S^{b}$ \\
\hline \multicolumn{6}{|c|}{ Reference } \\
\hline $\mathrm{H}$ & 27.28 & 0.03 & 0.00 & 0.00 & 0 \\
\hline $\mathrm{OH}$ & 22.23 & 15.48 & 0.00 & 0.02 & -0.0382 \\
\hline $\mathrm{OMe}$ & 21.31 & 19.05 & 0.07 & 2.99 & -0.0413 \\
\hline OEt & 21.1 & 19.86 & 0.06 & 3.41 & -0.0427 \\
\hline $\mathrm{NH}_{2}$ & 19.95 & 22.61 & 0.00 & 0.00 & -0.0711 \\
\hline \multicolumn{6}{|c|}{$\pi$-donor, $\sigma$-acceptor } \\
\hline $\mathrm{F}$ & 24.51 & 9.24 & 0.00 & 0.00 & -0.0098 \\
\hline $\mathrm{Cl}$ & 21.06 & 18.01 & 0.00 & 0.01 & -0.0222 \\
\hline $\mathrm{Br}$ & 20.07 & 21.78 & 0.00 & 0.03 & -0.0243 \\
\hline \multicolumn{6}{|c|}{$\pi$-acceptor } \\
\hline vinyl & 20.17 & 21.31 & 7.25 & 47.9 & -0.0863 \\
\hline \multicolumn{6}{|c|}{$\sigma$-donor } \\
\hline $\mathrm{Me}$ & 24.72 & 5.84 & 0.00 & 6.24 & -0.0184 \\
\hline t-butyl & 24.07 & 9.33 & 0.12 & 14.94 & -0.0169 \\
\hline TMS & 24.52 & 9.27 & 0.10 & 10.7 & -0.0145 \\
\hline \multicolumn{6}{|c|}{$\sigma$-acceptor } \\
\hline $\mathrm{CF}_{3}$ & 26.22 & 4.97 & 0.00 & 0.45 & 0.0031 \\
\hline
\end{tabular}

${ }^{a}\left[\sum n_{\text {a.o. }}^{2} / \sum n^{2}{ }_{\text {m.o. }}\right] \times 100=\%$ contribution. $\sum n_{\text {a.o. }}^{2}$ is the sum of the squares of the atomic orbital coefficients of the atom or group of interest and $\sum n^{2}{ }_{\text {m.o. }}$ is the sum of the squares of all atomic orbital coefficients in a specific molecular orbital. ${ }^{b}$ Change in spin at the oxygen atom (relative to $\mathrm{R}=\mathrm{H}$ ).

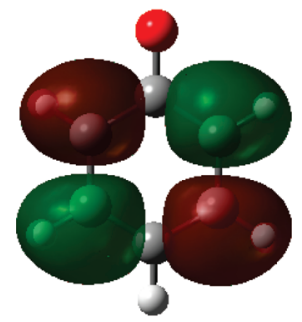

phenoxyl
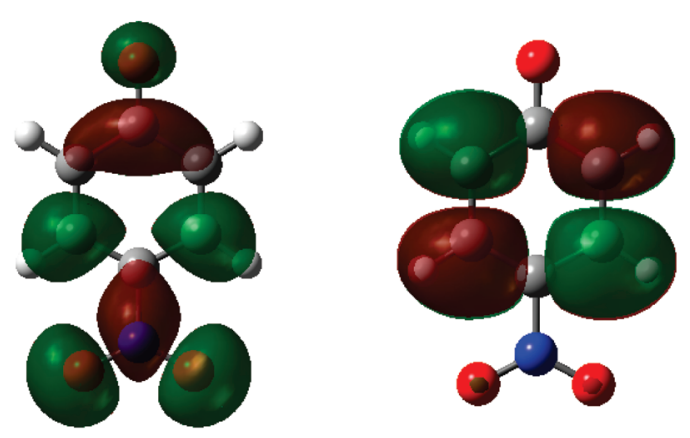

$\alpha$-LUMO

para- $\mathrm{NO}_{2}$-phenoxyl

$\alpha$-LUMO + 1

Figure 8. Comparison of the $\alpha$-LUMO orbitals for phenoxyl and $p-\mathrm{NH}_{2}$-phenoxyl radicals (top) with the LUMO and LUMO+1 orbitals of $p-\mathrm{NO}_{2}$-phenoxyl (bottom).

the contribution of $\mathrm{NO}_{2}$ to the HOMO is comparable to other substituents exhibiting similar degrees of spin polarization. In this regard, we believe that the model we have developed remains conceptually robust, but the HOMO-LUMO gap
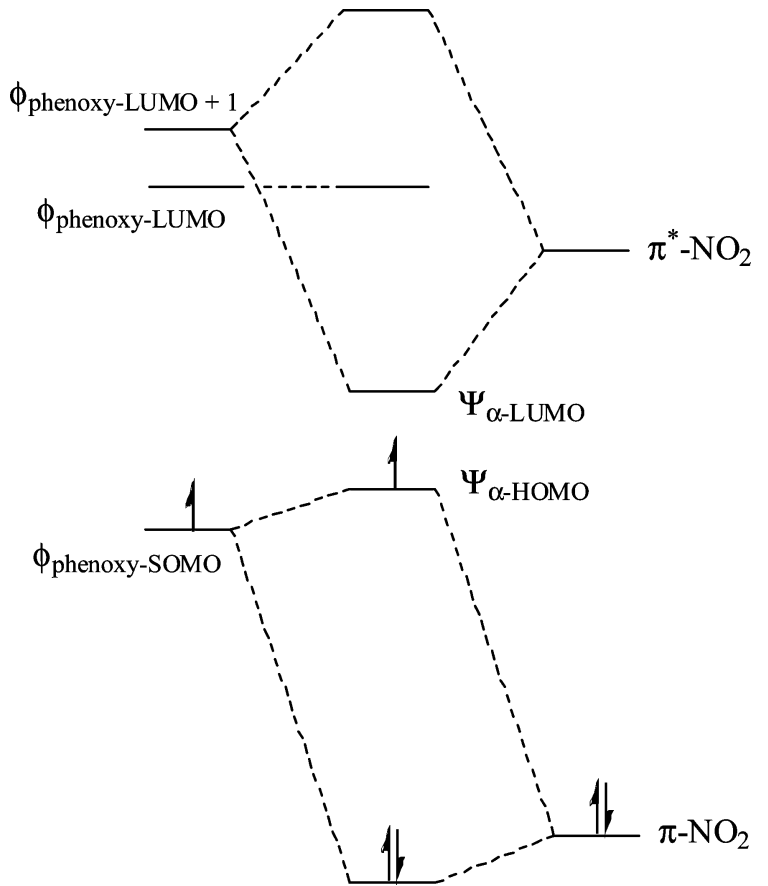

Figure 9. Proposed molecular orbital diagram for $p-\mathrm{NO}_{2}$-phenoxyl. The presence of a low-energy empty $\pi^{*}$ orbital on the $\mathrm{NO}_{2}$ group leads to a change in the nature of the LUMO and is responsible for the deviation exhibited by $p$ - $\mathrm{NO}_{2}$-phenoxyl from the correlation illustrated in Figure 5.

correlation breaks down in the case of $\mathrm{NO}_{2}$ due to the presence of a new interaction not present in the other compounds.

The vinyl group also shows significant contributions to the LUMO as well as exhibiting the same LUMO/LUMO+1 
inversion as seen for $p$ - $\mathrm{NO}_{2}$-phenoxyl. However, the situation for $p$-vinyl-phenoxyl is fundamentally different: whereas the $\mathrm{NO}_{2}$ adduct displays less spin polarization than the HOMOLUMO gap would suggest, the vinyl group appears to be just the opposite. At the same time, we note that the vinyl group is the most effective at polarizing unpaired spin away from the oxygen atom of any substituent we examined (Figure 1). This enhanced delocalizing ability of the vinyl group has also been mentioned by Henry et al. in a study of substituted methyl radicals. ${ }^{99}$ The contrast between $\mathrm{NO}_{2}$ and vinyl implies that $\alpha$-spin resonance delocalization coupled with $\beta$-spin donation is the best mechanism to delocalize spin, but the ability of $\mathrm{NO}_{2}$ to delocalize spin is attenuated compared to the vinyl group. This attenuation is most likely due to the inductive electron withdrawing ability of these substituents; it is known that an electron-withdrawing group usually plays a duel role in radical stability. ${ }^{100-105} \mathrm{~A}$ withdrawing group can stabilize a radical by resonance delocalization ( $\pi$-withdrawing) and at the same time destabilize the radical by electron induction ( $\sigma$-withdrawing). Destabilization by electron induction is not surprising due to the electron deficient nature of the oxygen radical center, and can be thought of as an unfavorable "pull-pull" dipole-dipole interaction. ${ }^{106}$ Therefore, we believe that the enhanced spin delocalization in the vinyl group can be linked to two contributing factors: (1) $\sigma$ donation by the vinyl group in the absence of inductive destabilization, the combination of which increases $\beta$-spin density at the oxygen center, and (2) stabilization of the radical by resonance delocalization of $\alpha$-spin density. The latter appears to be a particularly effective mechanism for delocalizing the spin in these systems.

Based on our analysis of $p$-vinyl-phenoxyl, we sought to identify even stronger spin delocalizing substituents. Toward this end, there are substituents known as "super radical stabilizers", such as $p-N=\mathrm{N}(\mathrm{O})-\mathrm{Bu}, p-N=\mathrm{N}-\mathrm{Ph}$, and $p-\mathrm{CH}=\mathrm{N}(\mathrm{O})-{ }^{\mathrm{t}} \mathrm{Bu} \cdot{ }^{107,108}$ Incorporation of the $\mathrm{CH}=\mathrm{N}(\mathrm{O}){ }^{\mathrm{t}} \mathrm{Bu}$ group at the para position of the phenoxy radical decreased unpaired spin density at the oxygen atom by $60 \%$ in part due to an increase in $\beta$-spin density at the oxygen atom, in line with our expectations based on the model just described.

$\sigma$-donors and $\sigma$-acceptors. The $\sigma$-donor substituents $\mathrm{CH}_{3}$, $t$-butyl, and TMS decrease the spin density at the oxygen atom, but to a lesser extent than that of the $\pi$-donors. This trend in spin delocalization is also mirrored in the larger HOMO-LUMO gaps for these substituents as compared to the $\pi$-donors. The delocalization of spin in these substituents can be accounted for by the hyperconjugation $\beta$-spin donation mechanism already discussed above and depicted in Scheme 3. A three-electron, two-orbital interaction can again be invoked to describe the spin delocalization in these substituents, where the interaction between a filled $\sigma$-orbital with the partially filled $\pi$-orbital of the phenoxy radical leads to a stabilizing interaction. ${ }^{97}$ The degree of stabilization between the substituents is small, and literature reports suggest that branching of the alkyl chain will lead to less effective hyperconjugation. ${ }^{69}$ This is exactly what is observed in the phenoxy radical complexes: the composition of the relevant molecular orbitals indicates delocalization of the wavefunction onto the substituent via mixing between the $\phi_{\text {phenoxy-somo }}$ and a filled $\sigma$-orbital of the $\sigma$-donor substituents.

With regard to $\sigma$-acceptor groups, studies by Brinck et al. ${ }^{71}$ as well as Bean ${ }^{72}$ indicate that $\mathrm{CF}_{3}$ is a radical destabilizing substituent which in the present context corresponds to increasing the spin density at the oxygen atom. Bean suggests this can be attributed to an anticaptodative effect arising from a "pull-pull" effect in the system due to decreased conjugation.
Brinck et al. points out that this substituent has been shown to be destabilizing in other radical delocalization scales such as $\sigma_{\alpha}{ }^{\circ}$ and $\sigma_{\mathrm{JJ}}{ }^{\circ}$. A similar mechanism is likely operative in $p$-Fphenoxyl, since $\sigma_{\mathrm{JJ}}{ }^{\circ}, \sigma_{\alpha}{ }^{\circ}$, and $\sigma_{\mathrm{C}}{ }^{\circ}$ have shown the para fluoro group to be destabilizing. It should be noted that, in the case of $\mathrm{CF}_{3}$, we observed a functional dependence as to whether $\mathrm{CF}_{3}$ increased or decreased spin density at the oxygen: calculations at the UBLYP/6-311G** level gave $\Delta S=-0.002$, whereas a value of $\Delta S=0.003$ was obtained with the hybrid UB3LYP functional. We are therefore reluctant to draw any definitive conclusions concerning the influence of $\sigma$-acceptors based on our calculations.

\section{Concluding Comments}

The goal of this study was to examine whether spin and charge density could be independently manipulated in a simple molecular system, and, more importantly, to understand the underlying mechanism for such a process. Using parasubstituted phenoxy radicals as a test case, we carried out calculations using a wide range of substituents. A strong correlation between the Hammett $\sigma_{\mathrm{p}}{ }^{+}$polar substituent constants and changes in charge density at the oxygen atom support previous work suggesting that resonance and inductive effects are largely responsible for charge polarization. Simple electronegativity arguments can be applied, wherein electron acceptors withdraw charge density and electron donors increase the charge density at the oxygen atom in the substituted phenoxy radicals. In contrast, the spin density at the oxygen atom behaved in a manner quite distinct from the charge; none of the commonly invoked spin polarization parameters were adequate for understanding our results. A correlation was established between the $\alpha$-HOMO- $\alpha$-LUMO gap and the spin density at the oxygen center whereby increasing/decreasing spin delocalization coincided with a decrease/increase in the $\alpha-\mathrm{HOMO}-\alpha-$ LUMO gap. A simple three-electron, two-orbital model was used to explain the correlation in which the amount of mixing between the $\phi_{\text {phenoxy-Somo }}$ and the $\phi_{\pi \text {-donor }}$ was identified as the dominating mechanism that controls both the HOMO-LUMO gap and the spin delocalization in these systems. $\pi$-acceptor substituents that were found to deviate from the correlation did so due to additional interactions from unoccupied $\pi^{*}$ orbitals of the substituent with the $\phi_{\text {phenoxy-LUMO+1 }}$, giving rise to a change in the character of the LUMO relative to other members of the series. Substituents that can serve as both a $\pi$-acceptor and a $\sigma$-donor (e.g., vinyl) were found to have the greatest propensity for redistributing spin density within the molecule.

Our results suggest that it should be possible to differentially polarize spin and charge through synthetic means by judicious choice of substituents. This could be of considerable use in the field of molecular magnetism, for example, whereby control of spin density at the radical centers could be used to manipulate intramolecular exchange interactions. One could also envision use of this approach in the design of catalysts for radical-radical coupling reactions, in which spatially distinct polarization of $\alpha$ - and $\beta$-spin density could facilitate the formation of $\sigma$ bonds. Efforts to implement some of the ideas generated from the results described in this report are ongoing.

Acknowledgment. The authors would like to thank Professors James E. Jackson and James F. Harrison for a number of fruitful discussions. This research was supported by the National Science Foundation (Grant No. CHE-0616340). 


\section{References and Notes}

(1) Wolf, S. A.; Awschalom, D. D.; Buhrman, R. A.; Daughton, J. M.; von Molnár, S.; Roukes, M. L.; Chtchelkanova, A. Y.; Treger, D. M. Science 2001, 294, 1488.

(2) Fert, A. Rev. Mod. Phys. 2008, 80, 1517

(3) Bogani, L.; Wernsdorfer, W. Nat. Mater. 2008, 7, 179.

(4) Hansch, C.; Leo, A.; Taft, R. W. Chem. Rev. 1991, 91, 165.

(5) Ewing, D. F. Correlation of NMR Chemical Shifts with Hammett $\sigma$ Values and Analogous Parameters. In Correlation Analysis in Chemistry: Recent Advances; Chapman, N. B., Shorter, J., Eds.; Plenum Press: New York, 1978.

(6) Yang, J. S.; Liu, K. T.; Su, Y. J. Phys. Org. Chem. 1990, 3, 723. (7) Pratt, D. A.; Dilabio, G. A.; Mulder, P.; Ingold, K. U. Acc. Chem. Res. 2004, 37, 334.

(8) Sekigawa, K. Tetrahedron 1972, 28, 505.

(9) Fernández, I.; Frenking, G. J. Org. Chem. 2006, 71, 2251.

(10) Wiberg, K. B. J. Org. Chem. 2002, 67, 4787.

(11) Liu, L.; Fu, Y.; Liu, R.; Li, R. Q.; Guo, Q. X. J. Chem. Inf. Comput. Sci. 2004, 44, 652.

(12) Krygowski, T. G.; Stêpień, B. T. Chem. Rev. 2005, 105, 3482.

(13) Genix, P.; Jullien, H.; Le Goas, R. J. Chemometrics 1996, 10, 631 .

(14) Head-Gordon, M. J. Phys. Chem. A 1996, 100, 13213.

(15) Koch, W.; Holthausen, M. C. A Chemist's Guide to Density Functional Theory; Wiley-VCH: Weinheim, Germany, 2000.

(16) Parr, R. G.; Yang, W. Density-Functional Theory of Atoms and Molecules; Clarendon: Oxford, 1989.

(17) Claiser, N.; Souhassou, M.; Lecomte, C.; Gillon, B.; Carbonera, C.; Caneschi, A.; Dei, A.; Gatteschi, D.; Bencini, A.; Pontillon, Y.; LelièvreBerna, J. Phys. Chem. B 2005, 109, 2723.

(18) Zheludev, A.; Barone, V.; Bonnet, M.; Delley, B.; Grand, A.; Ressouche, E.; Rey, P.; Subra, R.; Schweizer, J. J. Am. Chem. Soc. 1994, $116,2019$.

(19) McConnell, H. M. J. Chem. Phys. 1956, 24, 764.

(20) Remenyi, C.; Reviakine, R.; Kaupp, M. J. Phys. Chem. B 2007, 111,8290 .

(21) Nguyen, M. T.; Creve, S.; Vanquickenborne, L. G. J. Phys. Chem. A 1997, 101, 3174.

(22) Reiher, M. Faraday Discuss. 2007, 135, 97.

(23) Pillet, S.; Legrand, V.; Weber, H. P.; Souhassou, M.; Letard, J. F.; Guionneau, P.; Lecomte, C. Z. Kristallogr. 2008, 223, 235.

(24) Pierpont, C. G. Coord. Chem. Rev. 2001, 216-217, 99.

(25) Pierpont, C. G.; Buchanan, R. M. Coord. Chem. Rev. 1981, 38, 45 .

(26) Pierpont, C. G.; Lange, C. W. Prog. Inorg. Chem. 1994, 41, 331

(27) Kaim, W. Coord. Chem. Rev. 1987, 76, 187.

(28) Shultz, D. A.; Sloop, J. C.; Washington, G. J. Org. Chem. 2006, $71,9104$.

(29) Adam, W.; Borden, W. T.; Burda, C.; Foster, H.; Heidenfelder, T.; Heubes, M.; Hrovat, D.; Kita, F.; Lewis, S.; Scheutzow, D.; Wirz, J. J. Am. Chem. Soc. 1998, 120, 593.

(30) West, A. P., Jr.; Silverman, S. K.; Dougherty, D. A. J. Am. Chem. Soc. 1996, 118, 1452 .

(31) Geise, C. M.; Hadad, C. M. J. Org. Chem. 2000, 65, 8348.

(32) Mitani, M.; Yamaki, D.; Takano, Y.; Kitagawa, Y.; Yoshioka,

Y.; Yamaguchi, K. J. Chem. Phys. 2000, 113, 10486.

(33) Eaton, G. R.; Eaton, S. S. Acc. Chem. Res. 1988, 21, 107.

(34) Eaton, S. S.; Eaton, G. R. Coord. Chem. Rev. 1988, 83, 29.

(35) Shultz, D. A.; Bodnar, S. H.; Lee, H.; Kampf, J. W.; Incarvito,

C. D.; Rheingold, A. L. J. Am. Chem. Soc. 2002, 124, 10054.

(36) Shultz, D. A.; Fico, R. M., Jr.; Bodnar, S. H.; Kumar, R. K.; Vostrikova, K. E.; Kampf, J. W.; Boyle, P. D. J. Am. Chem. Soc. 2003, $125,11761$.

(37) Shultz, D. A.; Fico, R. M., Jr.; Lee, H.; Kampf, J. W.; Kirschbaum, K.; Pinkerton, A. A.; Boyle, P. D. J. Am. Chem. Soc. 2003, 125, 15426. (38) Shultz, D. A.; Sloop, J. C.; Coote, T.-A.; Beikmohammadi, M.;

Kampf, J.; Boyle, P. D. Inorg. Chem. 2007, 46, 273.

(39) Stone, T. J.; Waters, W. A. J. Chem. Soc. 1964, 213.

(40) Waters, W. A.; Stone, T. J. Proc. Chem. Soc. London 1962, 253.

(41) Dixon, W. T.; Norman, R. O. C. J. Chem. Soc. 1964, 4587.

(42) Scheffler, K.; Stegmann, H. B. Elektronenspinresonanz; Springer:

Berlin, 1970.

(43) Qin, Y.; Wheeler, R. A. J. Chem. Phys. 1995, 22, 1689.

(44) Wu, Y.; Lai, D. K. W. J. Org. Chem. 1996, 61, 7904.

(45) Kawai, A.; Hirakawa, M.; Abe, T.; Obi, K.; Shibuya, K. J. Phys. Chem. A 2001, 105, 9628.

(46) Broze, M.; Luz, Z. J. Chem. Phys. 1969, 51, 738.

(47) Broze, M.; Luz, Z. J. Chem. Phys. 1969, 51, 749.

(48) Hirota, N. J. Chem. Phys. 1962, 37, 1884.

(49) Frisch, M. J.; Trucks, G. W.; Schlegel, H. B.; Scuseria, G. E.; Robb, M. A.; Cheeseman, J. R.; Zakrzewski, V. G.; Montgomery, J. A., Jr.; Stratmann, R. E.; Burant, J. C.; Dapprich, S.; Millam, J. M.; Daniels,
A. D.; Kudin, K. N.; Strain, M. C.; Farkas, O.; Tomasi, J.; Barone, V.; Cossi, M.; Cammi, R.; Mennucci, B.; Pomelli, C.; Adamo, C.; Clifford, S.; Ochterski, J.; Petersson, G. A.; Ayala, P. Y.; Cui, Q.; Morokuma, K.; Malick, D. K.; Rabuck, A. D.; Raghavachari, K.; Foresman, J. B.; Cioslowski, J.; Ortiz, J. V.; Stefanov, B. B.; Liu, G.; Liashenko, A.; Piskorz, P.; Komaromi, I.; Gomperts, R.; Martin, R. L.; Fox, D. J.; Keith, T.; Al-Laham, M. A.; Peng, C. Y.; Nanayakkara, A.; Gonzalez, C.; Challacombe, M.; Gill, P. M. W.; Johnson, B.; Chen, W.; Wong, M. W.; Andres, J. L.; Gonzalez, C.; Head-Gordon, M.; Replogle, E. S.; Pople, J. A. Gaussian 98, revision A.4; Gaussian, Inc.: Pittsburgh, PA, 1998.

(50) Becke, A. D. Phys. Rev. A 1988, 38, 3098.

(51) Lee, C.; Yang, W.; Parr, R. G. Phys. Rev. B 1988, 37, 785.

(52) Becke, A. D. J. Chem. Phys. 1993, 98, 5648.

(53) Frisch, M. J.; Frisch, A. Gaussian 98 User's Reference; Gaussian, Inc.: Pittsburgh, 1998.

(54) Glendening, E. D.; Reed, A. E.; Carpenter, J. E.; Weinhold, F. NBO 3.1, Theoretical Chemistry Institute; University of Wisconsin: Madison, WI, 1996.

(55) SPARTAN, 5.0 ed.; Wavefunction Inc.: Irvine, CA, 1997.

(56) GaussView, 2.1 ed.; Gaussian, Inc.: Pittsburgh, PA, 2001.

(57) Baker, J.; Scheiner, A.; Andzelm, J. Chem. Phys. Lett. 1993, 216, 380 .

(58) Laming, G. J.; Handy, N. C.; Amos, R. D. Mol. Phys. 1993, 80, 1121.

(59) Pierpont, C. G.; Attia, A. S. Collect. Czech. Chem. Commun. 2001, $66,33$.

(60) Pierpont, C. G. Coord. Chem. Rev. 2001, 219, 415.

(61) Poddel'ski, A.; Cherkasov, V. K.; Abakumov, G. A. Coord. Chem. Rev. 2009, 253, 291.

(62) Merino, G.; Vela, A.; Heine, T. Chem. Rev. 2005, 105, 3812.

(63) Baerends, E. J.; Ellis, D. E.; Ros, P. Chem. Phys. 1973, 2, 41.

(64) Wiberg, K. B.; Rablen, P. R. J. Comput. Chem. 1993, 14, 1504.

(65) Brown, H. C.; Okamoto, Y. J. Am. Chem. Soc. 1958, 80, 4979.

(66) Correlation Analysis in Chemistry: Recent Advances; Plenum Press: New York, 1978.

(67) Shorter, J. Correlation analysis in oganic chemistry: an introduction to linear free-energy relationships; Oxford University Press: Oxford, 1973.

(68) Lind, J.; Shen, X.; Eriksen, T. E.; Merényi, G. J. Am. Chem. Soc. 1990, $112,479$.

(69) Wayner, D. D. M.; Arnold, D. R. Can. J. Chem. 1985, 63, 2378.

(70) Bauld, N. L. Radicals, Ion Radicals, and Triplets: The SpinBearing Intermediates of Organic Chemistry; Wiley-VHC, Inc.: Weinheim, Germany, 1997.

(71) Brinck, T.; Haeberlein, M.; Jonsson, M. J. Am. Chem. Soc. 1997, $119,4239$.

(72) Bean, G. P. Tertahedron 2002, 58, 9941.

(73) Viehe, H. G.; Janousek, Z.; Merényi, R.; Stella, L. Acc. Chem. Res. 1985, 18, 148.

(74) Viehe, H. G.; Merényi, R.; Stella, L.; Janousek, Z. Agnew. Chem. Int. Ed. Engl. 1979, 18, 917.

(75) Klessinger, M. Agnew. Chem. Int. Ed. Engl. 1980, 19, 908.

(76) Sustmann, R.; Korth, H.-G. Adv. Phy. Org. Chem. 1990, 26, 131.

(77) Dewar, M. J. S. J. Am. Chem. Soc. 1952, 74, 3353.

(78) Jiang, X.-K. Acc. Chem. Res. 1997, 30, 283.

(79) Jiang, X.; Ji, G. J. Org. Chem. 1992, 57, 6051.

(80) Dust, J. M.; Arnold, D. R. J. Am. Chem. Soc. 1983, 105, 1221.

(81) Creary, X. J. Org. Chem. 1980, 45, 280.

(82) Creary, X.; Mehrsheikh-Mohammadi, M. E.; McDonald, S. J. Org. Chem. 1987, 52, 3254 .

(83) Dincturk, S.; Jackson, R. A.; Townson, H.; Agribas, H.; Bellingham, N. C.; March, G. J. J. Chem. Soc. Perkin Trans. 2 1981, 1121.

(84) Jackson, R. A.; Dincturk, S. J. Chem. Soc. Perkin Trans. 2 1981, 1127.

(85) Fisher, T. H.; Meierhoefer, A. W. J. Org. Chem. 1978, 43, 221. 224.

(86) Fisher, T. H.; Meierhoefer, A. W. J. Org. Chem. 1978, 43,

(87) Amos, A. T.; Hall, G. G. Proc. R. Soc. Ser. A 1961, 263, 483.

(88) King, H. F.; Stanton, R. E.; Kim, H.; Wyatt, R. E.; Parr, R. G.

J. Chem. Phys. 1967, 47, 1936.

(89) Neese, F. J. Phy. Chem. Solids 2004, 65, 781.

(90) Ohta, K. J. Mol. Struct. (THEOCHEM) 2002, 587, 33.

(91) There has been some discussion about the possibility and usefulness of the Kohn-Sham orbitals (cf. 93-95) but good approximations to experimental excitation energies can be made from them (cf. 95).

(92) Stowasser, R.; Hoffmann, R. J. Am. Chem. Soc. 1999, 121, 3414.

(93) Bickelhaupt, F. M.; Baerends, E. J. Kohn-Sham Density Functional Theory: Predicting and Understanding Chemistry. In Reviews in Computational Chemistry; Lipkowitz, K. B., Boyd, D. B., Eds.; Wiley-VCH: New York, 2000; Vol. 15, pp 1-86.

(94) Chong, D. P.; Gritsenko, O. V.; Baerends, E. J. J. Chem. Phys. 2002, 116, 1760 

391.

(95) Savin, A.; Umrigar, C. J.; Gonze, X. Chem. Phys. Lett. 1998, 288,

(96) We stress that the straight line in Figure 5 is not meant to imply that spin density is necessarily a linear function of the HOMO-LUMO gap, merely that there is a correlation between the two variables.

(97) Bernardi, F.; Epiotis, N. D.; Cherry, W.; Schlegel, H. B.; Whangbo, M.; Wolfe, S. J. Am. Chem. Soc. 1976, 98, 469.

(98) Clark, T. Substituent Effects in Radical Chemistry. In Substituent Effects in Radical Chemistry; Viehe, H. G.; Janousek, Z.; Merenyi, R., Eds.; Reidel: Dordrecht, 1986; p 49.

(99) Henry, D. J.; Parkinson, C. J.; Mayer, P. M.; Radom, L. J. Phys. Chem. A 2001, 105, 6750.

(100) Cheng, J.; Liu, B.; Zhao, Y.; Wen, Z.; Sun, Y. J. Am. Chem. Soc. 2000, 122, 9987.
(101) Cheng, J; Zhao, Y. Tertahedron 1993, 49, 5267.

(102) Pasto, D. J.; Krasnansky, R.; Zercher, C. J. Org. Chem. 1987, 52, 3062.

(103) Fu, Y.; Liu, R.; Guo, Q.-X. J. Phys. Org. Chem. 2005, 18, 529.

(104) Bordwell, F. G.; Harrelson, J. A., Jr.; Zhang, X. J. Org. Chem. 1991, 56, 4448.

(105) Zipse, H. Top. Curr. Chem. 2006, 263, 163.

(106) Bordwell, F. G.; Zhang, X.-M.; Satish, A. V.; Chen, J.-P. J. Am. Chem. Soc. 1994, 116, 6605.

(107) Creary, X. Acc. Chem. Res. 2006, 39, 761

(108) Creary, X.; Engel, P. S.; Kavaluskas, N.; Pan, L.; Wolf, A. J. Org. Chem. 1999, 64, 5634.

JP905314H 